K. Heitmiller ${ }^{1}$, M. Innes ${ }^{2}$, V. Zollo ${ }^{3}$, C. Sansur ${ }^{4}$, R. Goldner 5 , D. Powell ${ }^{6}$, A. A. GASPARI ${ }^{1}$

\title{
Diagnostic dilemmas of Titanium Hypersensitivity in patients with medical implants: a case series
}

${ }^{1}$ Department of Dermatology and Cutaneous Biology, Thomas Jefferson University, Philadelphia, USA

${ }^{2}$ Department of Dermatology, University of Alabama, Alabama, USA

${ }^{3}$ Department of Dermatology, La Sapienza University, Rome, Italy

${ }^{4}$ Department of Neurosurgery, University of Maryland, Baltimore, Maryland

${ }^{5}$ Department of Dermatology, University of Maryland, Baltimore, Maryland

${ }^{6}$ Department of Dermatology, University of Utah, Salt Lake City, Utah

\section{KEY WORDS}

Titanium hypersensitivity; metal allergy; allergic contact dermatitis; metal implants; type I hypersensitivity; type IV hypersensitivity.

\author{
Corresponding author \\ Kerry Heitmiller \\ Department of Dermatology and Cutaneous Biology \\ Thomas Jefferson University \\ 833 Chestnut Street \\ 19107 Philadelphia, USA \\ E-mail: Kerry.heitmiller@jefferson.edu
}

Doi

10.23822/EurAnnACI.1764-1489.141

To the editor,

Titanium is used in numerous medical, dental devices and implants. It is considered a "hypoallergenic" alternative when there is concern for hypersensitivity to other metals (1-4). Nevertheless, titanium hypersensitivity $(\mathrm{TH})$ reactions have been reported in association with cardiac pacemakers and dental, cardiac, orthopedic, cardiovascular and neurosurgical implants (4-12). Metal allergy is traditionally considered a type IV hypersensitivity reaction. Type I hypersensitivity to metals is rare, but such reactions have been reported, including to titanium (13-16).

This case series highlights the diagnostic dilemmas associated with $\mathrm{TH}$ and emphasizes that a high index of suspicion for $\mathrm{TH}$ is required for prompt diagnosis and management of postoperative complications associated with $\mathrm{TH}$ reactions.
A retrospective review of four patient records with postoperative reactions following insertion of a titanium-containing implant was performed. The study complied with the ethical standards established in the Declaration of Helsinki of 1946.

\section{Case 1}

A 53-year-old African American female was referred for patch testing for suspected metal allergy to lumbar metal implants placed for degenerative disc disease. Three months after her procedure, she experienced worsening lower back pain, bilateral leg pain and paresthesias, as well as erythema, warmth and tenderness overlying the midline surgical scar. Lumbar computed tomography (CT) myelogram and magnetic resonance imaging (MRI) were unremarkable. Laboratory results including complete blood count, 
tissue cultures and erythrocyte sedimentation rate were also within normal limits. Synthes titanium alloy (TAN) rods and screws (titanium, 6\% aluminum, 7\% niobium and $0.02 \%$ nickel) had been used for the spinal fusion. Patch testing with the standard North American Contact Dermatitis Group (NACDG) standard series and Chemotechnique extended metal series was not tolerated due to an asthmatic reaction with facial angioedema within 24 hours of patch placement, requiring an emergency room visit for treatment with systemic corticosteroids. Limited patch tests to nickel and titanium were subsequently performed. Titanium nitride 5\% (Chemotechnique extended metal series) was placed on the patient's right forearm and nickel sulfate $2.5 \%$ (NACDG standard series) was placed on the left forearm. After 72 hours, the patient demonstrated a $1+$ reaction to nickel and no reaction to titanium; however, the patient reported a symptomatic reaction (pruritus) at the titanium patch test site prior to her visit. Thus, prick testing was also performed to assess immediate hypersensitivity to nickel and titanium. The skin on the right upper arm was pierced in three spots, patches were applied (nickel 2.5\%, titanium $10 \%$, and petrolatum, a control) and the patient was monitored for one hour. Urticarial reactions were observed at both the nickel and titanium sites, but not at the petrolatum control site. Prick testing of a healthy control to the same metals (nickel 2.5\%, titanium 10\%) was negative. The patient later disclosed a history of suspected metal allergy after a failed left foot bunionectomy required removal of titanium screws used during the procedure, although testing to evaluate for metal allergy had not been performed.

After confirming the presence of a metal allergy, the spinal implant was removed and replaced with bone morphogenic protein. Prior to wound closure, gram stain, cultures, and tissue samples from the wound and hardware were obtained and were negative. Pathology of the tissue samples revealed fragments of necrotic bone with inter-trabecular fibrosis and chronic lymphocytic inflammation, consistent with aseptic lymphocyte dominated vasculitis associated lesion (ALVAL, not shown). Following implant removal, the patient experienced resolution of her symptoms.

\section{Case 2}

A 42-year-old Caucasian female presented for evaluation for metal hypersensitivity reactions to cervical metal implants due to persistent neck pain and erythema of the anterior neck with delayed wound healing along the posterior incision site one year after the procedure. There was a history of multiple cervical spinal fusions/ reconstructions due to pseuduoarthrosis and hardware failure. CT of the cervical spine demonstrated lucency around the hardware, suggestive of hardware failure. Globus Coalition titanium alloy (TAV) implants (titanium, 6\% aluminum, and 4\% vanadium) had been used for the anterior cervical discectomy and fusion. Synthes titanium alloy (TAN) rods and screws (titanium, 6\% aluminum, $7 \%$ niobium and $0.02 \%$ nickel) were used for the posterior spinal fusion and a Synthes SynMesh vertebral body replacement device was inserted anteriorly. Patch testing was performed using solid metal samples (i.e. discs) of the Synthes plate and rod and the Globus plate. The discs were placed on the skin after cleaning with an alcohol pad and covered with cloth tape. At 24 and 48 hours, no reactions were observed. At 72 hours, few erythematous macules were observed under the Synthes and Globus plate samples. At one week, small, grouped, pruritic erythematous papules were observed under the Globus plate sample. No reaction was observed with the titanium rod sample. Patch testing with these plates on a healthy control did not result in any reactions. The allergy testing suggested a hypersensitivity to the Globus implant and the location of these implants appeared to be consistent with the anterior neck erythema the patient had developed postoperatively. The patient underwent removal of the Globus implants, which were replaced by structural allograft spacers, and recovered without complication.

\section{Case 3}

A 57-year-old Caucasian female was referred for evaluation for metal hypersensitivity to a cervical metal plate, which had been placed for degenerative disc disease. She developed erythema and edema of the face, neck, and upper chest shortly after the procedure, along with shortness of breath from a pleural effusion that began at the same time. Her symptoms did not resolve with antibiotics or systemic corticosteroids and she subsequently required multiple thoracenteses, which repeatedly revealed a highly eosinophilic transudate. The cervical plate used was a standard alloy composed of titanium, $6 \%$ aluminum and $4 \%$ vanadium (Ti6Al4V, TC4). Patch testing to NACDG standard series and Chemotechnique metal series showed only a positive $1+$ reaction to gold sodium thiosulfate. Prick testing to an arbitrarily selected titanium salt (titanium IV oxide 10\%/Chemotechnique) revealed an urticarial reaction within one hour. Repeat prick testing was performed using six titanium preparations (titanium IV oxide $0.1 \%$, titanium $1 \%$, titanium III nitride $5 \%$, titanium III oxalate decahydrate $5 \%$, titanium $10 \%$, titanium dioxide $10 \%$ ) as well as aluminum and vanadium. This revealed urticarial reactions to five of the six titanium salts (no reaction to titanium dioxide 10\%). Prick testing with these titanium salts in healthy controls $(\mathrm{N}=20)$ was negative. The patient was diagnosed with type I hypersensitivity to titanium and subsequently underwent removal of the plate in her neck with resolution of her symptoms within two months. The patient remains symptom free more than four years later.

\section{Case 4}

A 61-year-old Caucasian male presented for evaluation of a pruritic rash on his right knee (figure $\mathbf{1}$ a). The patient had developed the rash shortly after undergoing a right total knee replacement six months prior to presentation. He had been experiencing per- 
sistent right knee pain since the surgery, longer than the expected period of postoperative pain. A Triathlon Titanium implant (cementless) was used, a highly porous metal biologic fixation technology created using 3D-printing technology during which multiple layers of titanium powder are melted together. Physical exam demonstrated an ill-defined erythematous plaque localized around a well-healed linear scar on the right knee (figure $1 \mathbf{a}$ ). Patch testing to the NACDG standard series, Chemotechnique extended metal series, and dental series showed a positive +/reaction to titanium III oxalate decahydrate at 48 hours and $1+$ reaction at 72 hours (figure $\mathbf{1}$ b). The patient was diagnosed with $\mathrm{TH}$ secondary to recent implantation and was given triamcinolone $0.1 \%$ cream for symptomatic relief. The patient was counseled on the diagnosis and replacement of the implant was recommended. He declined revision of the knee replacement to avoid an additional surgical procedure. He has continued to experience chronic dermatitis with recurrent flares, controlled with topical corticosteroids, but has persistent knee pain.

We report four cases of suspected $\mathrm{TH}$, including two cases of type I hypersensitivity reactions. The clinical manifestations consistent with metal hypersensitivity, positive allergy testing, absence of a more likely diagnosis, and dramatic resolution of symptoms with explanation (in three of the four cases) support the diagnosis of TH. Additionally, for Case 1, there was peri-im- plant tissue for histologic study at the time of implant revision, which revealed the presence of ALVAL, one of the histologic biomarkers of metal hypersensitivity reactions $(17,18)$.

The prevalence of TH is difficult to assess due to lack of reliable detection methods; however, it is estimated to be 0.2 to $1 \%$ (16, 19, 20). In a study by Sicilia et al. (16), 35 patients at a dental clinic with symptoms consistent with $\mathrm{TH}$ and underwent patch testing and prick testing. The majority of patients with positive allergy testing had developed both type I and type IV hypersensitivity (positive patch and/or prick test) reactions to their titanium implants (16). The results of this study, in addition to the present cases, suggest $\mathrm{TH}$ reactions may be different compared to that of other metals.

While patch testing is considered the standard method for detecting metal allergy, its use for TH is not as well accepted (1-3). Positive patch test reactions to titanium are rare and a negative patch test may not exclude a diagnosis of TH. Current patch test formulations of titanium salts may not be adequate for the detection of type IV hypersensitivity to titanium because of low epidermal penetration $(19,21)$. Patients with a negative reaction to titanium on patch testing may still be diagnosed with TH based on positive reactions on other diagnostic tests and/or resolution of postoperative symptoms after removal of the titanium-containing implant $(1,3-5,7,9,12,14,15)$ Case 4 was the only patient who

Figure 1 - (a) Subacute dermatitis on the knee, adjacent to and overlying surgical scar seen in Case 4. (b) Patch test reaction to titanium (III) oxalate decahydrate at $72 \mathrm{~h}$, revealing a $1+$ reaction.

(a)

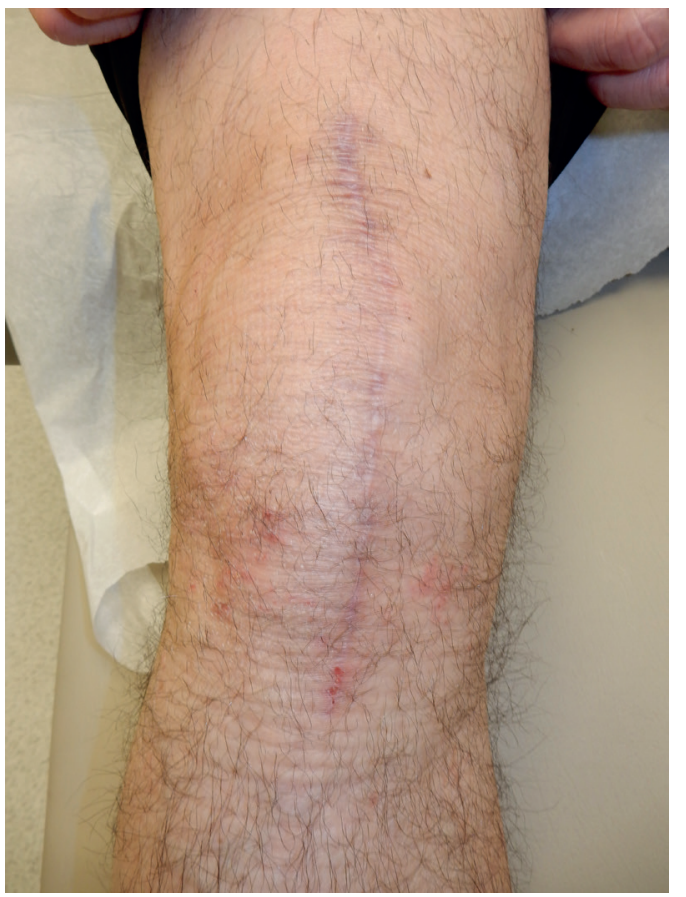

(b)

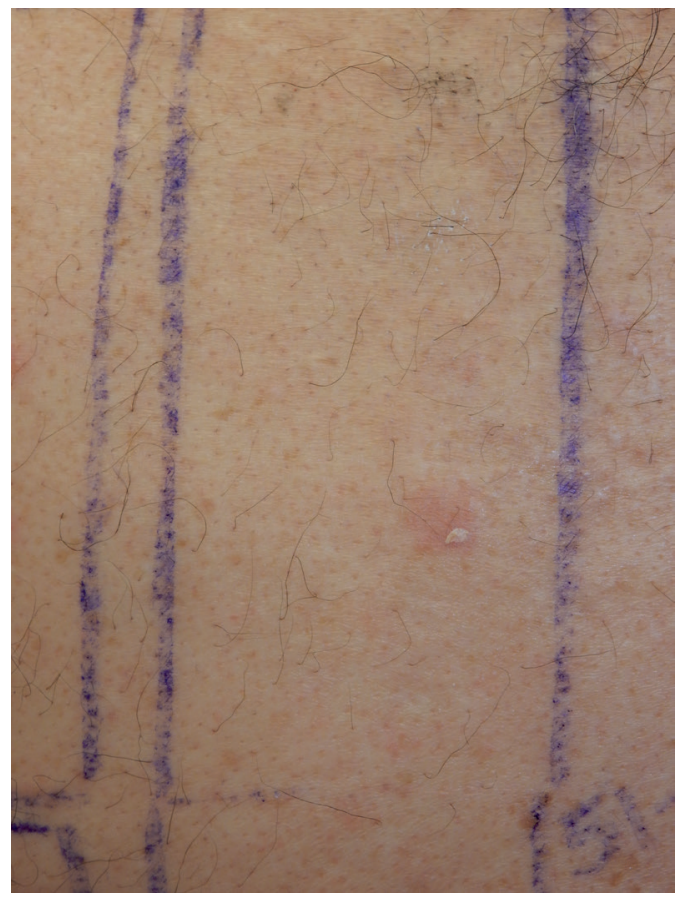


demonstrated a positive reaction to titanium with standard patch testing, allowing for the unambiguous diagnosis of TH. Case 2 was diagnosed via patch testing, although samples of the metal implant itself (discs) were used. The positive skin test to the disc and the resolution of signs and symptoms after removal suggest that the titanium allergy was relevant to the patient's favorable outcome. Testing with metal discs has been discouraged because of concerns about false negative patch testing (22). Despite these concerns, this patient exhibited a type IV hypersensitivity reaction to the disc, which was the basis for implant removal.

Thyssen et al. (23) proposed ten objective criteria that support implant-related metal hypersensitivity, four of which are considered major criteria: (1) eruption over the implant; (2) positive patch test to metal; (3) chronic dermatitis; (4) resolution of symptoms after removal of the implant. Three of the cases presented in this series did not fulfill all of the major criteria needed for the diagnosis of implant-related metal hypersensitivity, but, underwent implant removal with resolution of symptoms, supporting the diagnosis of TH. We suspect the patient in Case 4 would have also experienced improvement and/or resolution of symptoms with explantation, but the patient refused an additional procedure. Therefore, while these criteria are helpful in diagnosing implant-related metal hypersensitivity in general, they may or may not apply to $\mathrm{TH}$ and may be less sensitive for type I hypersensitivity.

This series adds to the literature on metal hypersensitivity reactions to titanium, suggesting that patch testing alone may be insufficient to confirm TH, and that prick testing should be considered to confirm the diagnosis of TH. We propose prick testing or alternative type I hypersensitivity testing methods for patients who present with type I hypersensitivity-like reactions or when patch testing is negative. As titanium presents unique challenges in patch testing, these additional testing methods may increase the sensitivity of allergy testing and allow for more reliable diagnosis.

\section{Conflict of interests}

The authors declare that they have no conflict of interests.

\section{References}

1. Wood MM, Warshaw EM. Hypersensitivity reactions to titanium: diagnosis and management. Dermatitis 2015;26(1):7-25.

2. Fage SW, Muris J, Jakobsen SS, et al. Titanium: a review on exposure, release, penetration, allergy, epidemiology and clinical reactivity. Contact Dermatitis 2016;74(6):323-345.

3. Muller K, Valentin-Thon E. Hypersensitivity to titanium: clinical and laboratory evidence. Neuroendocrinology Letters 2006;27:31-35.

4. Sakamoto K, Ando K, Noma D. Metal allergy to titanium bars after the Nuss procedure for pectus excavatum. Ann Thoracic Surg 2014;98:708-710.

5. Wang L-F, Wu J, Zheng C, et al. Long-term fever after hallux valgus surgery secondary to titanium allergy: a case report and review of the literature. The Journal of Foot \& Ankle Surgery 2016;55:12821286.

6. Hosoki M, Nishigawa K, Miyamoto Y, et al. Allergic contact dermatitis caused by titanium screws and dental implants. Journal of Prosthodontic Research 2016;60:213-219.

7. Yamauchi R, Morita A, Tsuji T. Pacemaker dermatitis from titanium. Contact Dermatitis 2000;42:52-53.

8. du Preez LA, Butow KW, Swart TJP. Implant failure due to titanium hypersensitivity/allergy? Report of a case. SADJ 2007;62(1):22-25.

9. Thomas P, Bandi WD, Maier S, et al. Hypersensitivity to titanium osteosynthesis with impaired fracture healing, eczema and T-cell hyperresponsiveness in vitro: case report and review of the literature. Contact Dermatitis 2006;55:199-202.

10. Hettige $S$, Norris JS. Mortality after local allergic response to titanium cranioplasty. Acta Neurochir 2012;154:1725-1726.

11. High WA, Ayers RA, Adams JR, et al. Granulomatous reaction to titanium alloy: an unusual reaction to ear piercing. J Am Acad Dermatol 2006;55(4):716-720.

12. Belohlavek J, Belohlavkova S, Hlubocky J, et al. Severe allergic dermatitis after closure of foramen ovale with Amplatzer occluder. Ann Thorac Surg 2013;96:e57-e59.

13. Walsh ML, Smith VH, King CM. Type 1 and type IV hypersensitivity to nickel. Australas J Dermatol 2010;51(4):285-286.

14. Willardson HB, Kobayashi TT, Arnold JG, et al. Diffuse urticarial reaction associated with titanium dioxide following laser tattoo removal treatments. Photomedicine and Laser Surgery 2017;35(3):176-180.

15. Sicilia A, Cuesta S, Coma G, et al. Titanium allergy in dental implant patients: a clinical study on 1500 consecutive patients consecutive patients. Clin Oral Impl Res 2008;19:823-835.

16. Saluja SS, Davis CL, Chong TA, et al. Contact urticaria to nickel: a series of 11 patients who were prick test positive and patch test negative to nickel sulfate $2.5 \%$ and $5.0 \%$. Dermatitis 2016;27(5):282287.

17. Wiley KF, Ding K, Stoner JA, et al. Incidence of pseudotumor and acute lymphocytic vasculitis associated lesion (ALVAL) reactions in metal-on-metal hip articulations: a meta-analysis. J Arthroplasty 2013;28(7):1238-45.

18. Willert HG, Buchhorn G, Fayyazi A. Histopathological changes in tissues surrounding metal/metal joints-signs of delayed hypersensitivity (DTH)? World Tribology Forum on Athroplasty 2001;147166.

19. Okamura T, Morimoto M, Fukushima D, et al. A Skin Patch Test for the Diagnosis of Titanium Allergy. Journal of Dental Research, 1999;78:1135.

20. Satapathy S, Satish Babu CL, Shetty $S$, et al. Titanium hypersensitivity: a clinical study. Int J Oral Implantol Clin Res 2014; 5(1):8-11.

21. Nakajima K. Study on patch test reagent for titanium. Kokubyo Gakkai zasshi. The Journal of the Stomatological Society 2007;74(2):92-98.

22. Heitmiller K, French A, Alaish SM, Goldner R, and Gaspari AA. An observational study of patch testing for metal Allergy with manufacturer-supplied materials before Nuss bar insertion. Dermatitis 2015;26(6):271-275.

23. Thyssen JP, Menné T, Schalock PC, et al. Pragmatic approach to the clinical work-up of patients with putative allergic disease to metallic orthopaedic implants before and after surgery. Br J Dermatol 2011;164:473-8. 